\section{Urine metabolomic analysis to detect metabolites associated with the development of contrast induced nephropathy}

\author{
Deborah B. Diercks ${ }^{1}$, Kelly P. Owen², Jeffrey A. Kline ${ }^{3}$, Mark E. Sutter ${ }^{2}$ \\ Department of Emergency Medicine, ${ }^{1}$ UT Southwestern Medical Center, Dallas, TX; ${ }^{2}$ Davis Medical Center, \\ University of California, Sacramento, $\mathrm{CA}^{3}{ }^{3}$ Indiana University, Indianapolis, IN, USA
}

Objective Contrast induced nephropathy (CIN) is a result of injury to the proximal tubules. The incidence of $\mathrm{CIN}$ is around $11 \%$ for imaging done in the acute care setting. We aim to analyze the metabolic patterns in the urine, before and after dosing with intravenous contrast for computed tomography (CT) imaging of the chest, to determine if metabolomic changes exist in patients who develop CIN.

Methods A convenience sample of high risk patients undergoing a chest CT with intravenous contrast were eligible for enrollment. Urine samples were collected prior to imaging and 4 to 6 hours post imaging. Samples underwent gas chromatography/mass spectrometry profiling. Peak metabolite values were measured and data was log transformed. Significance analysis of microarrays and partial least squares was used to determine the most significant metabolites prior to CT imaging and within subject. Analysis of variance was used to rank metabolites associated with temporal change and CIN. CIN was defined as an increase in serum creatinine level of $\geq 0.5$ $\mathrm{mg} / \mathrm{dL}$ or $\geq 25 \%$ above baseline within 48 hours after contrast administration.

Results We sampled paired urine samples from 63 subjects. The incidence of CIN was 6/63 (9.5\%). Patients without CIN had elevated urinary citric acid and taurine concentrations in the pre-CT urine. Xylulose increased in the post CT sample in patients who developed CIN.

Conclusion Differences in metabolomics patterns in patients who do and do not develop CIN exist. Metabolites may be potential early identifiers of CIN and identify patients at high-risk for developing this condition prior to imaging.

Keywords Metabolomics; Contrast; Nephropathy

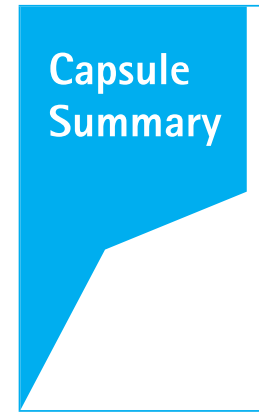

What is already known

Contrast induced nephropathy occurs in acute care and current diagnostic tools result in a delay in diagnosis.

What is new in the current study

Using urine metabolomics patterns, we were able to identify patients at risk for contrast induced nephropathy.
eISSN: 2383-4625

Received: 10 November 2015

Revised: 8 January 2016

Accepted: 1 February 2016

Correspondence to: Deborah B. Diercks Department of Emergency Medicine, UT Southwestern Medical Center, 5323 Harry Hines Boulevard, Dallas, TX 75390, USA

E-mail: Deborah.Diercks@ utsouthwestern.edu

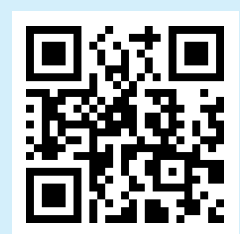

How to cite this article:

Diercks DB, Owen KP, Kline JA, Sutter ME. Urine metabolomic analysis to detect metabolites associated with the development of contrast induced nephropathy. Clin Exp Emerg Med 2016;3(2):204-212.

This is an Open Access article distributed under the terms of the Creative Commons Attribution Non-Commercial License (http:// creativecommons.org/licenses/by-nc/3.0/). 


\section{INTRODUCTION}

The use of computed tomography (CT) has increased over 200\% in the last decade.' Contrast induced nephropathy (CIN) that develops as a result of imaging using intravenous contrast enhancement, or other diagnostic procedures, has been reported to be the third leading cause of acute renal failure in hospitalized patients. It has been hypothesized that this occurs as a result of direct toxicity, oxidative stress, and ischemic injury. ${ }^{2}$ Numerous studies have evaluated the incidence of CIN in patients undergoing angiography. There are limited studies in the acute care setting; however, Mitchell et al.' reported the incidence of CIN in patients undergoing chest CT with contrast for the evaluation of pulmonary emboli to be close to $10 \%$. Studies have identified patient characteristics associated with the risk of developing $\mathrm{CIN}$, but there are limited diagnostic tools that can identify a patient at risk in the pre-CT or early post-CT time frame.' Therefore, a tool that could identify early risk factors for CIN would be valuable for patient care.

Metabolomic profiling is the identification of small molecule metabolites that are altered in response to injury. We have previously shown that urine metabolomic profiles differ in patients before and after intravenous contrast administration for CT scan. ${ }^{3}$ We hypothesize that metabolomic profiles will differ between those patients who develop CIN and those who do not after contrast administration. In addition we believe that metabolomics profiles prior to imaging may identify subjects who will go on to develop CIN and are therefore at higher risk. The specific aim of this pilot study is to determine if metabolomics profiles differ in patients who develop CIN after intravenous contrast administration for CT scan versus those who do not. Additionally, our goal was to identify specific urinary metabolites that warrant further investigation.

\section{METHODS}

This is a pilot study of prospectively identified patients undergoing a CT of the chest with intravenous contrast during their emergency department (ED) evaluation. The study was approved by the University of California, Davis institutional review boards.

\section{Study setting and selection of participants}

A convenience sample of patients was enrolled. To be eligible for the study, patients had to be $>18$ years old, undergoing $\mathrm{CT}$ angiography of the chest and have at least 1 of the following high risk features for $\mathrm{CIN}$ : diabetes, ${ }^{4,5}$ coronary artery disease, ${ }^{1}$ congestive heart failure, ${ }^{4,6}$ chronic kidney disease (baseline creatinine
$>1.5 \mathrm{mg} / \mathrm{dL}$ or glomerular filtration rate $<60 \mathrm{~mL} / \mathrm{min} / 1.73 \mathrm{~m}^{2}$ ). Past medical history was confirmed by chart review if available, or patient report. In addition, patients must have been given a physician assessment of $>75 \%$ likelihood of hospital admission.

Patients were excluded from the study if they had an estimated glomerular filtration rate $<15 \mathrm{~mL} / \mathrm{min} / 1.73 \mathrm{~m}^{2}$, a history of organ transplantation, were currently on immunosuppressive medications, were septic or on antibiotic therapy, had a history of or were currently receiving dialysis of any type, had an exposure to iodinated contrast within 3 days prior to the study, had more than one contrast CT ordered, or had multiple doses of contrast given.

Patients were managed according to the treating provider recommendations. No intervention was requested as part of this study. There was no institutional standard for mandatory fluid administration or use of $\mathrm{N}$-acetylcysteine prior to $\mathrm{CT}$ scanning.

\section{lodinated contrast}

All patients received approximately $120 \mathrm{~mL}$ of intravenous iodinated contrast material (iohexol; Omnipaque 350, GE Healthcare, Chicago, IL, USA) that was administered via computer-controlled automated power injector at $4 \mathrm{~mL}$ per second.

\section{Data collection and processing}

ED data were collected prospectively after patients were identified as fulfilling inclusion and exclusion criteria, and informed consent was obtained. Data collection included race, demographics, dietary history, medical history, physical examination, and electrocardiogram findings, as documented by the treating emergency physician. Medical history was confirmed through patient self-report and review of the medical record when available. Medications administered in the ED and before arrival were also recorded. Final ED diagnosis was based on the treating physician's impression. No additional laboratory tests were mandated as a part of the trial study and the treating physician ordered all tests, except urinary metabolomics analysis, according to their clinical judgment. Chest radiography findings documented by a board-certified radiologist, and laboratory tests, were obtained from the medical record. Urine samples were collected as a midstream sample or via a foley bag prior to CT scan and 4 to 6 hours post imaging. Samples were aliquoted into $2 \mathrm{~mL}$ samples and frozen at $-80^{\circ} \mathrm{C}$.

All patients were followed by chart review throughout their index stay to document in-hospital events. Serum creatinine levels were recorded at presentation, and at 24 and 48 hours.

The outcome measure was the presence $\mathrm{CIN}_{1}$ which was defined as an increase in serum creatinine level of $\geq 0.5 \mathrm{mg} / \mathrm{dL}$ or $\geq 25 \%$ above baseline within 48 hours after contrast administra- 
tion. For labeling purposes, patients who developed CIN are hereafter referred to as cases and patients without CIN are controls.

\section{Gas chromatography mass spectometry analysis}

Urine samples required no preparation prior to freezing. Neat urine samples were lyophilized without further pretreatment. Twenty microliter of $40 \mathrm{mg} / \mathrm{mL}$ methoxylamine hydrochloride in pyridine was added to the dried samples, and samples were agitated at $30^{\circ} \mathrm{C}$ for $30 \mathrm{~min}$. Subsequently, $180 \mu \mathrm{L}$ of trimethylsilylating agent $\mathrm{N}$-methyl-N-trimethylsilyltrifluoroacetamide was added, and samples were agitated at $37^{\circ} \mathrm{C}$ for 30 minutes. Gas chromatography mass spectometry analysis was performed using a Agilent $6890 \mathrm{~N}$ gas chromatograph (Agilent Technologies, Atlanta, GA, USA) interfaced to a time-of-flight Pegasus III mass spectrometer (Leco, St. Joseph, MI, USA).$^{7-9}$ Automated injections were performed with a programmable robotic Gerstel MPS2 multipurpose sampler (Mülheim an der Ruhr, Germany). Initial peak detection and mass spectrum deconvolution was performed with ChromaTOF software ver. 2.25 (Leco), and later samples were exported to the netCDF format for further data evaluation with MZmine and XCMS. To be considered a known metabolite, molecular compounds must be identified with $>50 \%$ certainty. If this degree of certainty was unobtainable, identified compounds were given the name "unknown", followed by a numeric number.

\section{Statistical analysis}

Continuous data was presented as medians and interquartile range. The statistical analysis was performed on (natural) log-transformed data that was range scaled (mean-centered and divided by the range of each variable) to adjust for concentration differences of metabolites in the sample. The primary objective was to identify metabolomics differences based on the presence of CIN post CT scan. Transformed data were plotted to assess for normality.

\section{Pre-CT analysis}

A t-test was performed to determine differences in the metabolites, with a P-value threshold of $<0.05$. Partial least squares-discriminate analysis (PLS-DA) was used to identify metabolites associated with CIN (class difference). The features were ranked based on their regression coefficients and plotted by their variable of importance in projection score. The variable of importance in projection score is a weighted sum of squares and takes into the account the amount of explained $Y$-variation in each dimension. Significance analysis of microarrays (SAM) is designed to address the false discovery rate when running multiple tests on high-dimensional microarray data. SAM assigns a significance score to each variable based on its change relative. The optimal delta was selected based on the target identification of 10 significant metabolites. The false discovery rate is the proportion of the metabolite change attributed to chance alone.

\section{Post imaging analysis}

Univariate analysis methods are the most common methods used for exploratory data analysis. For two- factor data, the basic approach is two-way analysis of variance (ANOVA). In time series, data samples are measured from the same subjects from different time points and therefore within subject ANOVA was used. Analysis was performed in Metaboanalyst 2.0 (Edmonton, Alberta, Canada). ${ }^{10}$

Multivariate empirical Bayes time-series analysis (MEBA) was developed for analysis of microarray time-course data and allows grouping by a pre-specified condition. MEBA compares the profiles of the different time series of the metabolites. The outcome is a ranked list of all metabolites that show differences in their temporal profile between the experimental groups. Analysis was performed in Metaboanalyst 2.0.

\section{RESULTS}

A total of 100 subjects were consented. Thirty-seven were ex-

Table 1. Demographics and patient characteristics

\begin{tabular}{lc}
\hline Variable & Value \\
\hline Age (yr) & $54(50-66)$ \\
Male & $31(49.2)$ \\
Caucasian race & $35(55.6)$ \\
Smoker (current) & $18(28.6)$ \\
Heart failure & $21(33.3)$ \\
Diabetes & $26(41.3)$ \\
Hypertension & $42(66.7)$ \\
Coronary artery disease & $16(25.8)$ \\
Heart rate (bpm) & $84(73-109)$ \\
Systolic blood pressure (mmHg) & $136(119-157)$ \\
Serum creatinine (mg/dL) & $0.94(0.74-1.14)$ \\
Serum BUN (mg/dL) & $13(10-18)$ \\
Final diagnosis & \\
Chest pain-not otherwise specified or ACS & $22(34.9)$ \\
Heart failure & $11(17.5)$ \\
Pneumonia & $8(12.7)$ \\
Asthma/COPD & $4(6.4)$ \\
Muskuloskeletal & $4(6.4)$ \\
Pulmonary emboli & $3(4.8)$ \\
Syncope & $2(3.2)$ \\
Arrhythmia & $2(3.2)$ \\
Other (unknown, mass, etc.) & $6(11.1)$ \\
Hours from CT to urine collection & $4.63(4-7)$ \\
\hline & \\
\hline
\end{tabular}

Values are presented as median (interquartile range) or number (\%). BUN, blood urea nitrogen; ACS, acute coronary syndrome; COPD, chronic obstructive pulmonary disease; $\mathrm{CT}$, computed tomography. 
cluded due to lack of pre and post urine samples or lack of 24hour creatinine value. The cohort comprised 63 patients, 31 were

Table 2. Important metabolites identified by t-test pre-CT

\begin{tabular}{llll}
\hline & P-value & $-\log 10$ & FDR \\
\hline Uracil & 0.00044094 & 3.3556 & 0.038075 \\
Uric acid & 0.00054007 & 3.2676 & 0.038075 \\
N-acetyllysine & 0.0026186 & 2.5819 & 0.12307 \\
Tyrosine & 0.0036236 & 2.4409 & 0.12773 \\
Ribose & 0.0057628 & 2.2394 & 0.12982 \\
Glycine & 0.0059732 & 2.2238 & 0.12982 \\
Citric acid & 0.0064451 & 2.1908 & 0.12982 \\
4-Hydroxymandelic acid & 0.0076673 & 2.1154 & 0.13514 \\
Ethanolamine & 0.010374 & 1.9841 & 0.16252 \\
N-acetylaspartic acid & 0.014264 & 1.8458 & 0.20112 \\
Galactinol & 0.025339 & 1.5962 & 0.25063 \\
Lysine & 0.026522 & 1.5764 & 0.25063 \\
Beta-alanine & 0.028332 & 1.5477 & 0.25063 \\
Glycolic acid & 0.028472 & 1.5456 & 0.25063 \\
2,3-Dihydroxybutanoic acid NIST & 0.028855 & 1.5398 & 0.25063 \\
Alpha ketoglutaric acid & 0.031523 & 1.5014 & 0.25063 \\
Lauric acid & 0.037764 & 1.4229 & 0.25063 \\
Xylulose NIST & 0.039411 & 1.4044 & 0.25063 \\
Glycocyamine & 0.043575 & 1.3608 & 0.25063 \\
Pseudo uridine & 0.043869 & 1.3578 & 0.25063 \\
N-acetyl-glutamic acid & 0.044078 & 1.3558 & 0.25063 \\
Alanine & 0.04412 & 1.3554 & 0.25063 \\
Urea & 0.044171 & 1.3549 & 0.25063 \\
5-Hydroxymethyl-2-furoic acid NIST & 0.045294 & 1.344 & 0.25063 \\
\hline
\end{tabular}

$\mathrm{CT}$, computed tomography; FDR, false discovery rate; NIST, National Institute of Standards and Technology.

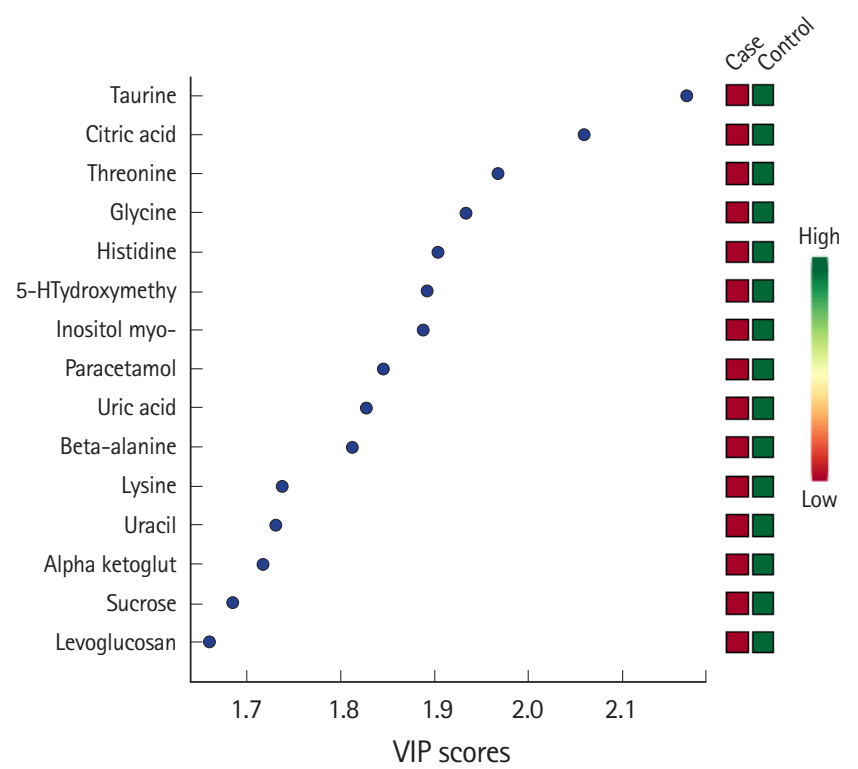

Fig. 1. Partial least squares analysis pre- computed tomography urine with variable importance in projection (VIP) scores and association with the development of contrast induced nephropathy (case). males (49.2) and the median age was 54 years (interquartile range 50 to 66). Of the 63 subjects, 6 met the criteria for CIN (cases), leaving 57 controls. The most common reported final ED diagnosis was chest pain not otherwise specified/acute coronary syndrome (Table 1).

\section{Pre-CT urine analysis}

In the pre-CT urine analysis, we identified nine metabolites associated with the outcome measure with a P-value $<0.01$ (Table 2). Of these nine, uric acid and uracil were the most significant. PLSDA ranked the most significant metabolites associated with the cases or controls. This analysis showed that higher citric acid and taurine levels were associated with controls (Fig. 1).

Uracil and uric acid were also identified metabolites associated with CIN in the SAM analysis (Table 3 and Fig. 2). Differences between cases and controls of the most significant peak metabolites found in the SAM analysis and PLS-DA are presented in Fig. 3.

Table 3. Significance analysis of microarrays significant metabolites

\begin{tabular}{llccc}
\hline & D-value & $\begin{array}{c}\text { Standard } \\
\text { deviation }\end{array}$ & $\begin{array}{c}\text { Unadjusted } \\
\text { P-value }\end{array}$ & Q-value \\
\hline Uracil & 3.7487 & 0.36036 & 0.00078014 & 0.012017 \\
Uric acid & 3.684 & 0.38714 & 0.00085106 & 0.012017 \\
N-acetyllysine & 3.1585 & 0.28349 & 0.0032624 & 0.027467 \\
Tyrosine & 3.0446 & 0.31453 & 0.0042553 & 0.027467 \\
Ribose & 2.8777 & 0.40455 & 0.0057447 & 0.027467 \\
Glycine & 2.8645 & 0.52686 & 0.0060284 & 0.027467 \\
Citric acid & 2.8366 & 0.56668 & 0.0068085 & 0.027467 \\
4-Hydroxymandelic acid & 2.7721 & 0.38061 & 0.0082979 & 0.029291 \\
\hline
\end{tabular}

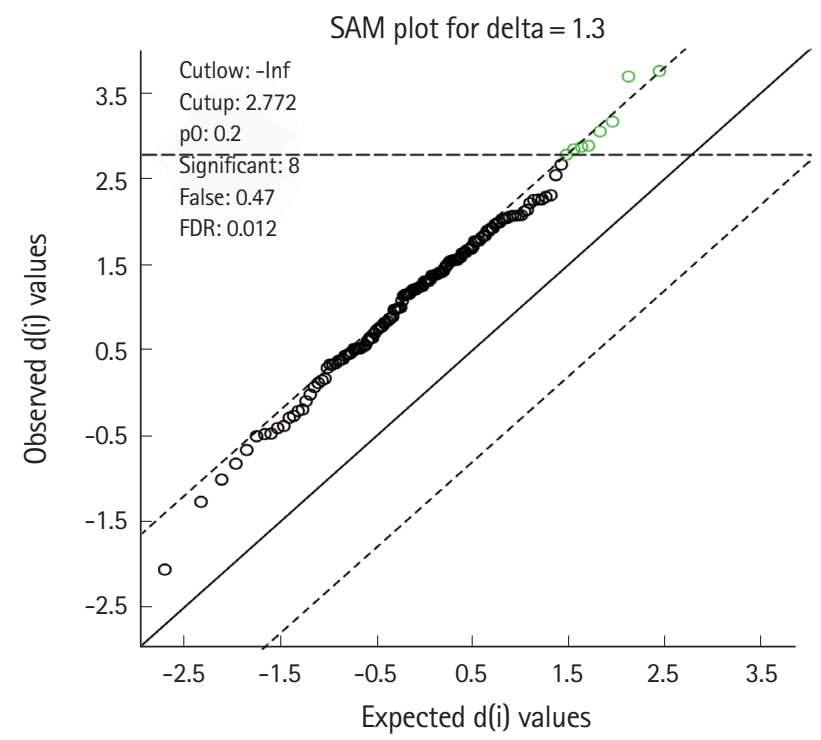

Fig. 2. Significance analysis of microarrays (SAM) shows 8 significant metabolites identified in the pre-computed tomography urine sample associated with contrast induced nephropathy. These are presented in Table 3. FDR, false discovery rate. 

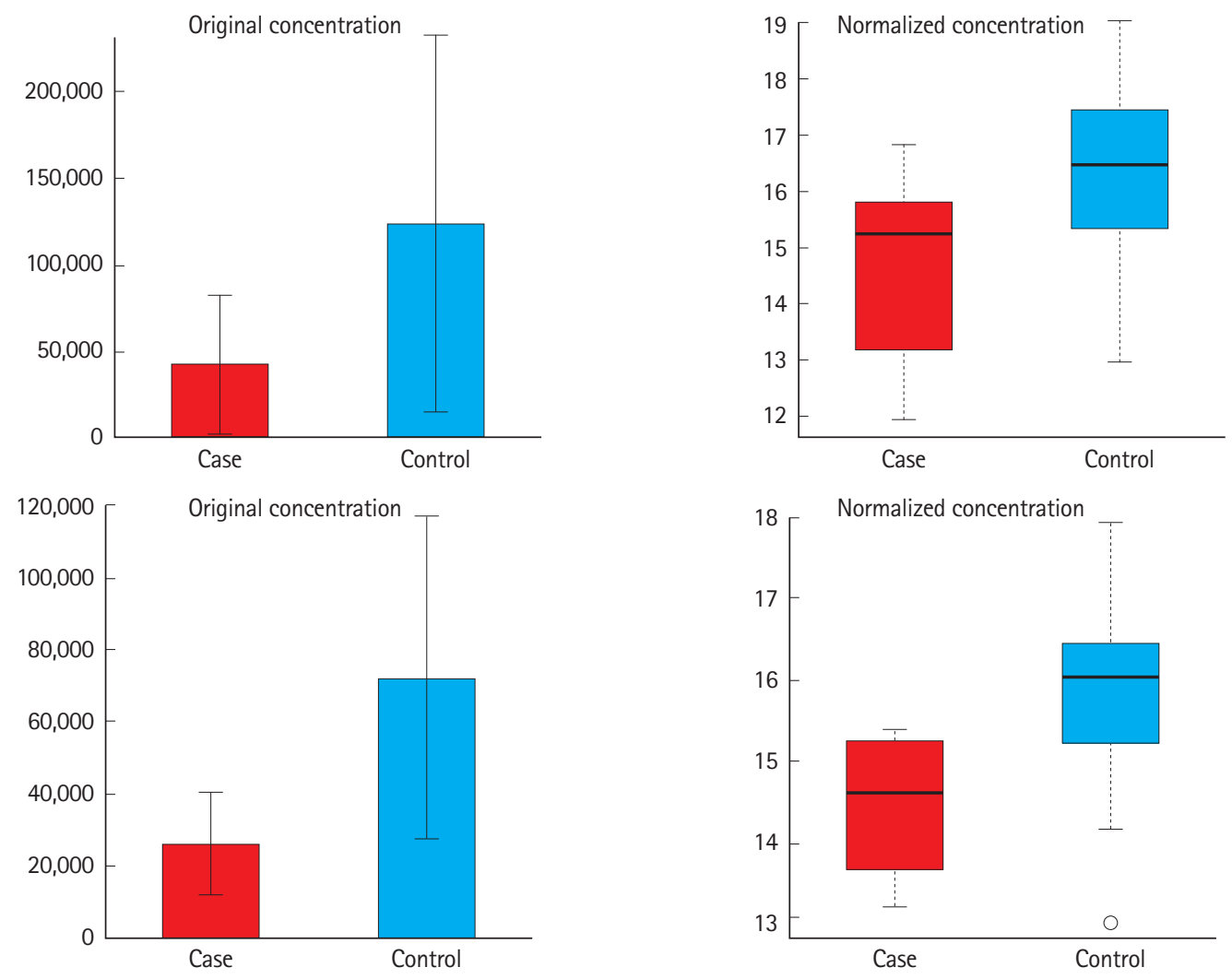

B
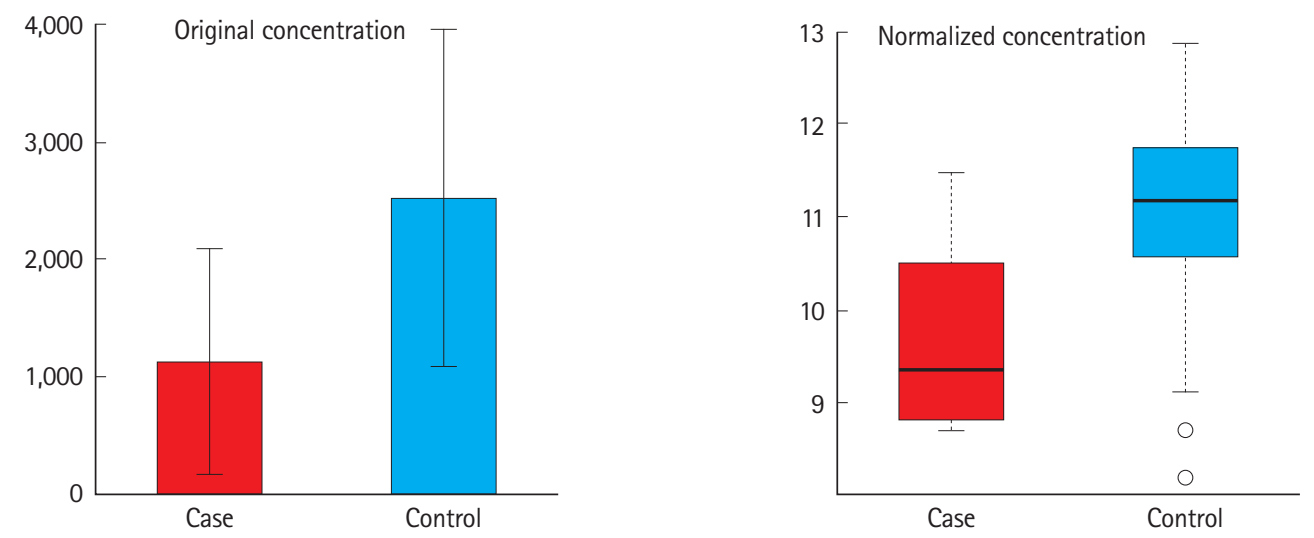

C
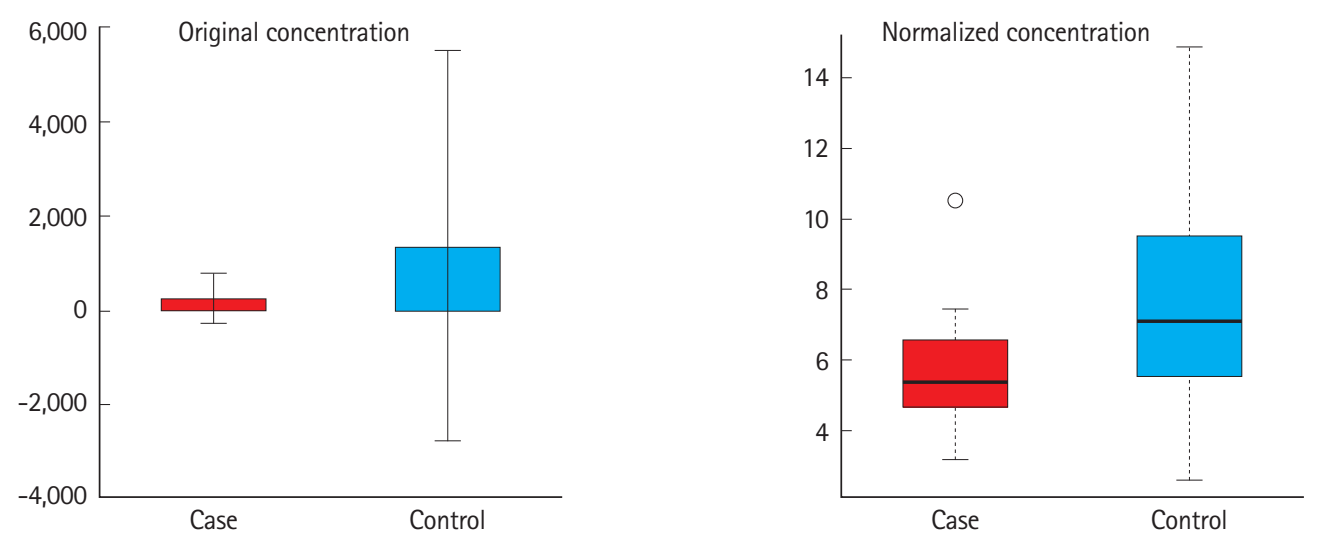

D

Fig. 3. Box plots of metabolites presented as adjusted and log transformed comparing patients with contrast induced nephropathy (case) and without (control). (A) Citric acid, (B) uric acid, (C) uracil, and (D) taurine. 

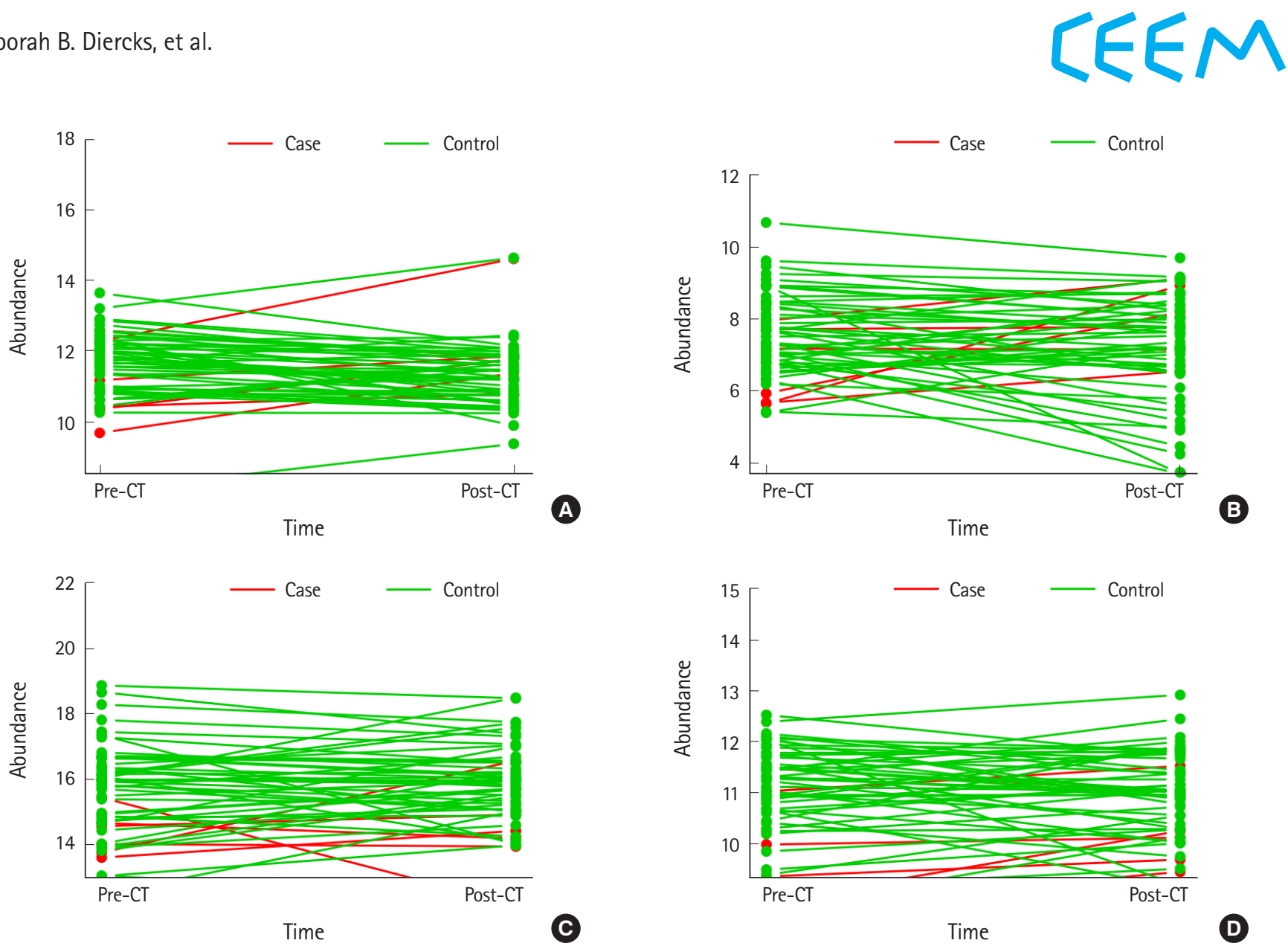

Fig. 4. Top 4 metabolites in the Multivariate Bayesian time-series analysis in post-imaging urine. (A) Threonic acid, (B) xylonic acid, (C) glycine, and (D) uracil. CT, computed tomography.

\section{Post-CT analysis}

The 4 top metabolites ranked showing temporal differences between cases and controls are xylonic acid, glycine, uracil and threonic acid (Fig. 4). Using ANOVA within a subject, xylulose was the most important metabolite (Table 4).

\section{DISCUSSION}

Renal function has been noted to become altered in up to $4 \%$ to $20 \%$ of all patients in hospitalized patients. ${ }^{11-13}$ Contrast-induced nephropathy is a leading cause of acute renal failure in this hospitalized patient population. ${ }^{14}$ Because of the late rise in serum creatinine, there is a temporal delay in identification of injury. In this study we did find differences in metabolomics profiles in those who would go on to develop CIN compared to those who did not develop CIN. Additionally, specific metabolites were significantly different between patients developing CIN and those that did not. When pre-contrast urine was analyzed concentrations of citric acid and taurine were higher in those that did not develop $\mathrm{CIN}$. When evaluating urine metabolite changes pre and post CT, xylulose, glycine, uric acid, and threonic acid were significant.
This study suggests that changes in the urine metabolomics profile of patients with CIN occur, and identifies metabolites for further evaluation in the development of CIN after CT imaging of the chest with contrast. The mechanisms behind the development of CIN are complex. They include potential interplay of vasoconstriction, oxidative stress, and direct tubular toxicity leading to hypoxia of the outer medulla. ${ }^{15-21}$ Studies have shown that the contrast administration not only results in vasoconstriction, but toxicity results in proximal tubularvacuolar transformation, interstitial edema and tubular degeneration. ${ }^{22}$ In addition changes to the proximal tubules result in alterations in electrolyte homeostasis. Given the complex pathophysiology behind the renal effects of intravenous contrast, multiple markers may identify inju$r y$, as different effects occur on urinary enzymes and tubular location. ${ }^{23,24}$

Given the numerous pathologic mechanisms behind the development of $\mathrm{CIN}$, it is not surprising that multiple statistically significant metabolites were identified. Several of these identified metabolites either mitigate oxidative damage or are a marker of an oxidative stress. Our results showed that subjects with higher levels of urea developed more CIN whereas higher levels of citric 
Table 4. Significant variables in the within subject ANOVA analysis

\begin{tabular}{lclc}
\hline & Phenotype & \multicolumn{1}{c}{ Time } & Interaction \\
\hline Xylulose NIST & 0.3629 & $1.70 \mathrm{E}-05$ & 0.96624 \\
Succinic acid & 0.6543 & 0.046879 & 0.59449 \\
Galactinol & 0.71555 & 0.044967 & 0.37627 \\
Levoglucosan & 0.72951 & 0.033927 & 0.37627 \\
Pinitol NIST & 0.87585 & 0.044698 & 0.50307 \\
Pyrogallol & 0.94838 & 0.00037119 & 0.37627 \\
Glycerol-3-galactoside & 0.94838 & 0.0079989 & 0.37627 \\
Phenol & 0.94838 & 0.0082768 & 0.68288 \\
UDP-glucuronic acid & 0.94838 & 0.026679 & 0.37627 \\
Quinic acid & 0.94838 & 0.026679 & 0.37627 \\
Pimelic acid & 0.94838 & 0.026679 & 0.41876 \\
Sucrose & 0.94838 & 0.044698 & 0.37627 \\
1-Desoxypentitol NIST & 0.94838 & 0.044698 & 0.93117 \\
2,5-Furandicarboxylic acid NIST & 0.98932 & 0.0082768 & 0.37627 \\
7-Methylguanine NIST & 0.98932 & 0.044698 & 0.99737 \\
3,4-Dihydroxyphenylacetic Acid & 0.9955 & 0.0018637 & 0.14347 \\
Catechol & 0.9955 & 0.036242 & 0.68288 \\
Furoylglycine NIST & 0.99802 & 0.01496 & 0.6106 \\
\hline
\end{tabular}

ANOVA, analysis of variance; NIST, National Institute of Standards and Technology; UDP, uridine diphosphate.

acid and taurine developed less.

As damage to the kidney occurs, proteins (containing nitrogen) are excreted. ${ }^{22}$ Both citric acid and taurine possess anti-oxidant properties and taurine has been shown to attenuate CIN in a canine model. ${ }^{25}$ Additionally, when evaluating metabolites with significant changes between pre and post intravenous contrast, $x y-$ lulose and threonic acid were significant. Xylulose is a pentose sugar that oxidizes to form xylonic acid. ${ }^{26}$ Our results showed that Xylonic acid levels rose post intravenous contrast demonstrating the process of oxidation. Similar to xylonic acid, threonic acid is the metabolic breakdown product of the anti-oxidant ascorbic acid (vitamin C), and both L-xylulose and ascorbic acid arise from chemical reduction of glucuronic acid. ${ }^{27}$ The presence and increasing concentrations of both of these acids after contrast exposure suggests initial reductive stress followed by an oxidative burst, possibly as a result of renal cortical vasospasm resulting in a hypoxia-reoxygenation pattern in the kidney medulla induced by contrast exposure in the kidney. ${ }^{28-30}$

Unlike our findings of increasing levels of xylonic acid and threonic acid after contrast exposure, we did identify that concentrations of uracil and glycine changed but not in a uniform direction. Attempting to determine a specific metabolic function involving uracil is challenging since it is one of the four nucleotides that make up RNA and thus its presence is ubiquitous. On the other hand, our identification of glycine as a potential marker for CIN is consistent with previous animal models. ${ }^{31-33}$ These animal studies showed a protective effect of glycine from both oxidant stress as well as cellular hypoxia. ${ }^{31,32}$ Cellular hypoxia may be a result of changes in renal blood flow in response to contrast administration.

\section{Limitations}

This is a pilot trial that was underpowered to detect an association of specific metabolites with CIN but rather our focus was to identify if metabolomic differences occur in patients with CIN and identify metabolites that warrant further investigation. In addition, due to the small sample size, we were unable to adjust for underlying medical illnesses, time since last meal, medications, or baseline renal function. Some urine samples were collected outside the target range due to inability to void. However these were the first urine samples available from the patients. This study did not address the clinical relevance of CIN. Specific isomers of metabolites were not defined.

\section{Summary and clinical implications}

$\mathrm{CIN}$ is a well described complication of intravenous contrast. Earlier identification of patients at risk may be possible through the identification of metabolite patterns that are highly associated with the disease, and it may improve the diagnosis. In this study we have shown that there are differences in urine metabolites between patients who do and do not develop CIN, prior to contrast administration and that changes in certain metabolites over time are associated with the diagnosis.

\section{CONFLICT OF INTEREST}

No potential conflict of interest relevant to this article was reported.

\section{ACKNOWLEDGMENTS}

Funding for this project was obtained through a grant from the UC Davis Department of Emergency Medicine.

\section{REFERENCES}

1. Mitchell AM, Kline JA. Contrast nephropathy following computed tomography angiography of the chest for pulmonary embolism in the emergency department. J Thromb Haemost 2007;5:50-4.

2. Messana JM, Cieslinski DA, Humes HD. Comparison of toxicity of radiocontrast agents to renal tubule cells in vitro. Ren Fail 1990;12:75-82.

3. Diercks DB, Owen KP, Tolstikov V, Sutter ME, Kline JA. Urinary 
metabolomic analysis to detect changes after intravenous, non-ionic, low osmolar iodinated radiocontrast for computerized tomographic imaging. West J Emerg Med 2014;15:152-7.

4. Mehran R, Nikolsky E. Contrast-induced nephropathy: definition, epidemiology, and patients at risk. Kidney Int Suppl 2006;100:S11-5.

5. Bartholomew BA, Harjai KJ, Dukkipati S, et al. Impact of nephropathy after percutaneous coronary intervention and a method for risk stratification. Am J Cardiol 2004;93:1515-9.

6. Freeman RV, O'Donnell M, Share $D$, et al. Nephropathy requiring dialysis after percutaneous coronary intervention and the critical role of an adjusted contrast dose. Am J Cardiol 2002; 90:1068-73.

7. Weckwerth W, Loureiro ME, Wenzel K, Fiehn O. Differential metabolic networks unravel the effects of silent plant phenotypes. Proc Natl Acad Sci U S A 2004;101:7809-14.

8. Nikiforova VJ, Kopka J, Tolstikov V, et al. Systems rebalancing of metabolism in response to sulfur deprivation, as revealed by metabolome analysis of Arabidopsis plants. Plant Physiol 2005;138:304-18.

9. Schad M, Mungur R, Fiehn O, Kehr J. Metabolic profiling of laser microdissected vascular bundles of Arabidopsis thaliana. Plant Methods 2005;1:2.

10. Xia J, Mandal R, Sinelnikov IV, Broadhurst D, Wishart DS. MetaboAnalyst 2.0: a comprehensive server for metabolomic data analysis. Nucleic Acids Res 2012;40:W127-33.

11. Liss $P$, Nygren $A$, Olsson U, Ulfendahl HR, Erikson U. Effects of contrast media and mannitol on renal medullary blood flow and red cell aggregation in the rat kidney. Kidney Int 1996;49: 1268-75.

12. Rauch $D$, Drescher $P$, Knes JM, Madsen PO. Variable effects of iodinated contrast media on different rabbit arteries in vitro. Urol Res 1997;25 Suppl 1:S21-3.

13. Wang YX, Jia YF, Chen KM, Morcos SK. Radiographic contrast media induced nephropathy: experimental observations and the protective effect of calcium channel blockers. Br J Radiol 2001;74:1103-8.

14. McCullough PA, Wolyn R, Rocher LL, Levin RN, O'Neill WW. Acute renal failure after coronary intervention: incidence, risk factors, and relationship to mortality. Am J Med 1997;103: 368-75.

15. Rauch D, Drescher P, Pereira FJ, Knes JM, Will JA, Madsen PO. Comparison of iodinated contrast media-induced renal vasoconstriction in human, rabbit, dog, and pig arteries. Invest Radiol 1997;32:315-9.

16. Heyman SN, Brezis M, Epstein FH, Spokes K, Silva P, Rosen S. Early renal medullary hypoxic injury from radiocontrast and indomethacin. Kidney Int 1991;40:632-42.

17. Parvez Z, Patel NB, Nelson JE. Urinary adenosine deaminase binding protein, a marker of contrast media induced acute renal damage. Clin Chim Acta 1990;190:111-3.

18. Jakobsen JA. Renal effects of iodixanol in healthy volunteers and patients with severe renal failure. Acta Radiol Suppl 1995;399:191-5.

19. Jakobsen JA, Berg KJ, Brodahl U, Laake B, Moxness A. Renal effects of nonionic contrast media after cardioangiography. Acta Radiol 1994;35:191-6.

20. Jakobsen JA, Nossen JO, Jorgensen NP, Berg KJ. Renal tubular effects of diuretics and X-ray contrast media. A comparative study of equimolar doses in healthy volunteers. Invest Radiol 1993;28:319-24.

21. Kaneko K, lkebe A, Shimazaki S, Yabuta K. Differences in renal tubular toxicity of high- and low-osmolality contrast media. Nephron 1989;51:579-80.

22. Thomsen HS, Dorph $\mathrm{S}$, Larsen $\mathrm{S}$, et al. Urine profiles and kidney histology after ionic and nonionic radiologic and magnetic resonance contrast media in rats with cisplatin nephropathy. Acad Radiol 1995;2:675-82.

23. Tumlin J, Stacul F, Adam A, et al. Pathophysiology of contrastinduced nephropathy. Am J Cardiol 2006;98:14K-20K.

24. Wright PA. Nitrogen excretion: three end products, many physiological roles. J Exp Biol 1995;198(Pt 2):273-81.

25. Hizoh I, Strater J, Schick CS, Kubler W, Haller C. Radiocontrastinduced DNA fragmentation of renal tubular cells in vitro: role of hypertonicity. Nephrol Dial Transplant 1998;13:911-8.

26. Pezzotti F, Therisod M. Enzymatic synthesis of aldonic acids. Carbohydr Res 2006;341:2290-2.

27. Englard S, Seifter $S$. The biochemical functions of ascorbic acid. Annu Rev Nutr 1986;6:365-406.

28. Palm F, Carlsson PO, Hansell $P$, Hellberg 0 , Nygren A, Liss $P$. Altered response in renal blood flow and oxygen tension to contrast media in diabetic rats. Acta Radiol 2003:44:347-53.

29. Weisberg LS, Kurnik PB, Kurnik BR. Radiocontrast-induced nephropathy in humans: role of renal vasoconstriction. Kidney Int 1992;41:1408-15.

30. Zhang $Y$, Wang J, Yang $X$, et al. The serial effect of iodinated contrast media on renal hemodynamics and oxygenation as evaluated by ASL and BOLD MRI. Contrast Media Mol Imaging 2012;7:418-25.

31. Weinberg JM, Davis JA, Abarzua M, Rajan T. Cytoprotective effects of glycine and glutathione against hypoxic injury to renal tubules. J Clin Invest 1987;80:1446-54.

32. Weinberg JM, Buchanan DN, Davis JA, Abarzua M. Metabolic aspects of protection by glycine against hypoxic injury to iso- 
lated proximal tubules. J Am Soc Nephrol 1991;1:949-58.

33. Weinberg JM, Davis JA, Abarzua M, Kiani T, Kunkel R. Protection by glycine of proximal tubules from injury due to inhibi- tors of mitochondrial ATP production. Am J Physiol 1990; 258(6 Pt 1):C1127-40. 